\title{
Towards Supporting Provenance Gathering and Querying in Different Database Approaches
}

\author{
Flavio Costa ${ }^{1(凶)}$, Vítor Silva ${ }^{1}$, Daniel de Oliveira ${ }^{2}$, \\ Kary A.C.S. Ocaña ${ }^{1}$, and Marta Mattoso ${ }^{1}$ \\ ${ }^{1}$ COPPE, Federal University of Rio de Janeiro, Rio de Janeiro, Brazil \\ \{flscosta, silva,kary, marta\}@cos.ufrj.br \\ ${ }^{2}$ Fluminense Federal University, Niteroi, Brazil \\ danielcmo@ic.uff.br
}

\begin{abstract}
The amount of provenance data gathered from Scientific Workflow Management Systems (SWfMS) and stored in databases has been growing considerably. Some difficulties are related to representation, access and query provenance databases. Despite the effort of PROV W3C group, data analyses may require different strategies of query specification because of the volume of data to be analyzed and the nature of queries. Another important point is the new approaches to store and retrieve provenance, some technologies are more appropriate than others. However, when applications are tightly coupled to specific technologies, it is difficult to take advantage of innovation. Based on these issues, we have built WfP-API, an API to store and perform queries in different provenance databases.
\end{abstract}

\section{Introduction}

Due to specific characteristics of Scientific Workflow Management Systems (SWfMS), experiments can generate provenance data in many different ways, in many different formats or even in many different repositories $[1,2]$. To diminish the difficulties to deal with provenance coming from different repositories, W3C defined the PROV data model, which is currently a recommendation [3]. To represent typical properties on provenance from scientific workflows, we proposed PROV-Wf [4], an specialization of PROV. PROV-Wf represents provenance data gathered from scientific workflow executions to be queried during or after execution. We implemented PROV-Wf in a relational database, enabling the analysis of provenance data in a well-structured way with SQL. However, the interaction of scientists with PROV-Wf databases is typically through an interface with parameterized pre-defined relational queries. In fact, there is no consensus on the best representation to deal with provenance and database systems can vary in performance significantly even within the same data schema representation. For example, different database indexing and storage approaches may present different

The research presented in this paper was partially funded by CNPq and FAPERJ grants. 
performance characteristics to execute provenance queries [5]. We can also mention that learning new database semantics for querying provenance is not an easy activity, especially for a non-specialist in computer science. In this paper, we propose WfP-API as an alternative way to SWfMS to have more flexibility when storing and querying provenance data. The API aims at storing provenance data in a variety of databases without having to concern about database system specific features. Our contribution is towards a PROV-Wf - based API, to be invoked from SWfMS, which is agnostic to the underlying database system. The API allows for querying provenance associated with workflow systems using one data model.

\section{WfP-API: Storing and Querying Workflow Provenance Data}

WfP-API is a provenance API that queries provenance databases based on the elements of PROV-Wf data schema [4]. WfP-API has two layers: WfP Object Layer and WfP Connector Layer. The former layer represents scientific workflows using adapted elements from PROV-Wf. In this first version, WfP Object Layer does not represent the agent elements (Scientist and Machine), while the software agent (Program) and Execute activity (PROV core structure of activity) are represented within the Activation concept. WfP Object Layer relies on object-oriented model to ease the integration with SWfMS to perform queries in DBMS. WfP Connector Layer establishes a connection between WfP-API object representation and DBMS. For each DBMS, a new instance of the connector has to be implemented. WfP-API currently supports Neo4J DBMS. Users of WfP-API need to choose the type of database to define connectors, while invocation to WfP Object layer is the same for every database connector.

\section{Implementation and Final Remarks}

WfP-API was developed according to the class diagram of Fig. 1. The dark gray classes describe each element of a scientific workflow (defined by WfP Object Layer) and scientists manipulate properties by calling getters and setters methods, such as getStartTime (that returns the activity start time) method in Activity class. Light gray classes represent internal controllers to define workflow structure (Object class) and connect WfP-API and the developed queries for a specific DBMS system (through Connector class). According to the specified database system, WfP Connector layer instantiates an object to use the DBMS, such as Neo4J Connector class. This class implements every abstract method from Connector class, with respect to the DBMS query language. Users may also need other queries than defined by WfP-API. In this case, they have to define their queries using the workflow object representation and extend connectors of the chosen DBMS.

Finally, the white classes represent DBMS connectors to the WfP-API. For example, for Neo4J DBMS we developed a Neo4JConnector class, which presents methods to establish a connection with this system (start method), to close this connection (close method), to determine workflow structure (storeActicity method), to 
store provenance data at runtime (updateRunningActivations method) or to execute custom queries developed by users (getWorkflowExecutionTime method).

WfP-API represents provenance based on the PROV-Wf data model, which follows W3C PROV recommendations. Furthermore, specialists only need to learn workflow semantics, since database semantics are already defined in WfP Connector Layer. Preliminary results showed that WfP-API was able to perform several queries that allow for steering of scientific workflow executions.

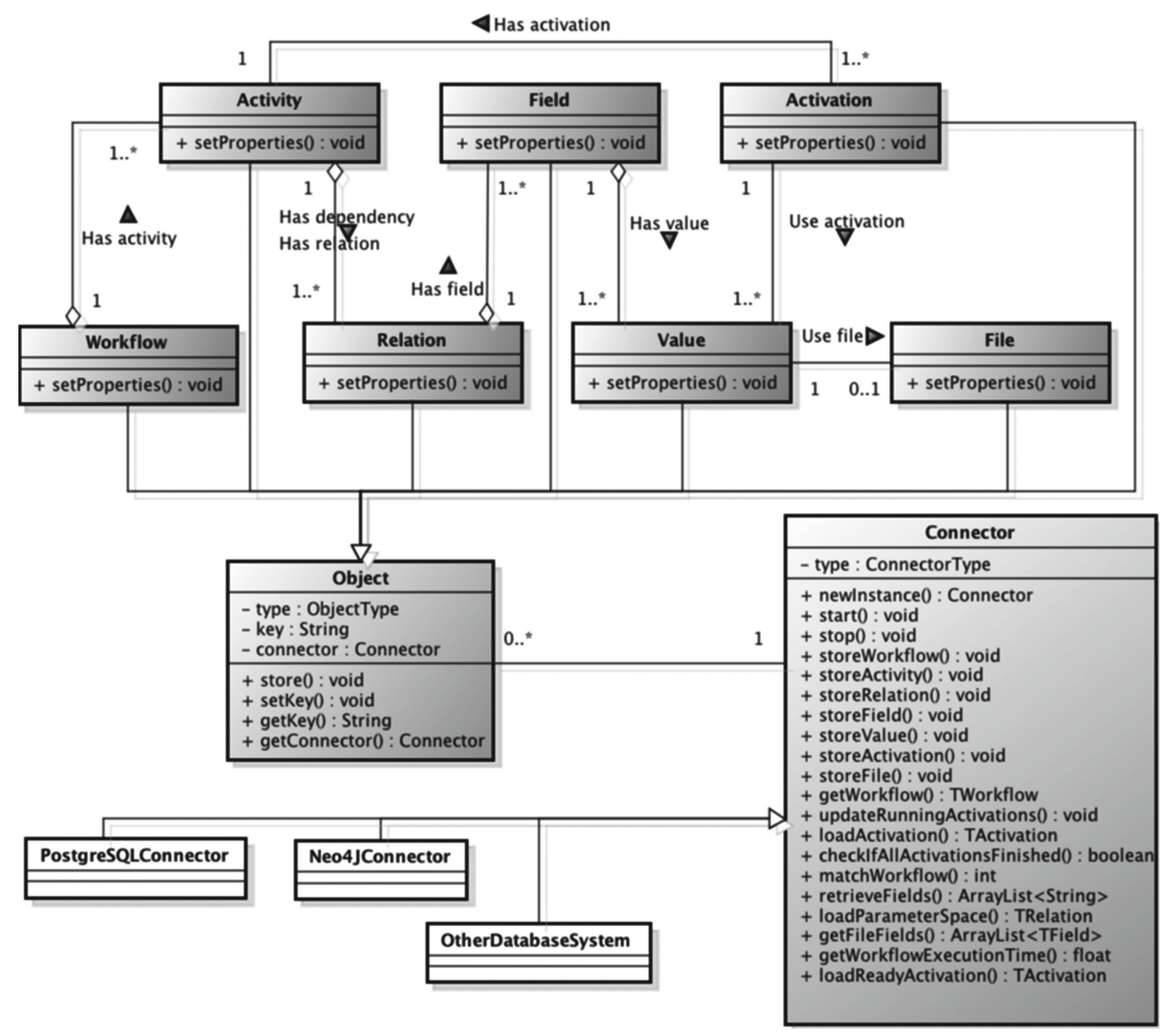

Fig. 1. Class diagram of WfP-API

\section{References}

1. Chirigati, F., Freire, J.: Towards integrating workflow and database provenance. In: Groth, P., Frew, J. (eds.) IPAW 2012. LNCS, vol. 7525, pp. 11-23. Springer, Heidelberg (2012)

2. Allen, M.D., Chapman, A., Blaustein, B., Seligman, L.: Getting it together: enabling multiorganization provenance exchange. In: TaPP (2011)

3. Missier, P., Belhajjame, K., Cheney, J.: The W3C PROV family of specifications for modelling provenance metadata. In: EDBT/ICDT 2013, pp. 773-776. ACM, New York, NY, USA (2013) 
4. Costa, F., Silva, V., De Oliveira, D., Ocaña, K., Ogasawara, E., Dias, J., Mattoso, M.: Capturing and querying workflow runtime provenance with PROV: a practical approach. In: EDBT/ICDT 2013 Workshops, pp. 282-289. ACM, New York, NY, USA (2013)

5. Muniswamy-Reddy, K.-K.: Deciding how to store provenance. Technical report 03-06, Computer Science, Harvard University (2006) 